\title{
SLAM BASED ON INFORMATION FUSION OF STEREO VISION AND ELECTRONIC COMPASS
}

\author{
Junmin Li, ${ }^{*}$ Jinge Wang, ${ }^{*}$ Simon X. Yang, ${ }^{* *}$ and Shiwei Jia*
}

\begin{abstract}
Due to the effects of image resolution, camera calibration and work environment in stereo vision-based robot's SLAM (Simultaneous Localization and Mapping), there are certain problems in location accuracy, robustness and anti-jamming capability. Meanwhile, because pose estimation is achieved through incremental iteration, there is also cumulative error. In this paper, an integrated location algorithm based on stereo vision and electronic compass is proposed to improve accuracy and robustness through information fusion. The rotation angles obtained by electronic compass and stereo vision respectively are fused by an improved fuzzy adaptive extended Kalman filter. Then initial pose estimation is obtained by the rotation angles and 3D coordinates of the time $t$ and the time $t+1$, and accurate pose estimation is realized by an adaptive particle filter. Finally, the map is updated by a Kalman filter. Experiment results show that the location accuracy, robustness and real-time performance are better than stereo vision alone and the traditional fuzzy adaptive extended Kalman filter.
\end{abstract}

\section{Key Words}

SLAM, information fusion, stereo vision, electronic compass

\section{Introduction}

SLAM is one of the most challenging open problems for developing truly autonomous robots. It can be stated as the problem of a robot building a map of an unknown environment while simultaneously tracking its position using the partially built map. Vision systems have acquired growing importance in mobile robotics during the past years due to their low cost and rich information that cameras provide in comparison with traditional robotic sensors, like laser scanners or sonars. Liang employed a novel detection algorithm based on a visual dictionary [1]. First, features in each image are described by the SURF (Speed Up Robust Feature)

* School of Mechanical Engineering, Xihua University, China; e-mail: Lijunmin1975@163.com, wangjg@mail.xhu.edu.cn, jiasw@nercita.org.cn

** Advanced Robotics and Intelligent Systems Laboratory, School of Engineering, University of Guelph, Guelph ON N1G 2W1, Canada; e-mail: syang@uoguelph.ca

Recommended by Prof. Chaomin Luo

(DOI: 10.2316/Journal.206.2016.3.206-4748) method. Then, these visual features are classed into visual words based on a fuzzy C-means algorithm. Saeedi proposed the approach of multiple-robot SLAM with a novel occupancy grid map fusion algorithm [2]. Map fusion is achieved through a multi-step process that includes image preprocessing, segmentation and cross-correlation.

In order to improve mobile robot intelligence and the ability to adapt to unknown complex environments, multisensor information fusion technology has become one of the key technologies and a hot research in the field. Practice has proved that fusion technology can greatly improve its robustness and stability [3]-[5]. The common methods of information fusion are as follows: the weighted average method, Bayes method, Kalman filter, D-S evidence synthesis method, fuzzy inference, neural networks and rough set method [6]-[13]. According to the disadvantages of the above methods, we present a new information fusion algorithm of an improved fuzzy adaptive extended Kalman filtering (FA-EKF) in this paper, which can fuzzily adjust the weights of electronic compass's noise and stereo vision's noise through the on-line monitoring of innovation mean and covariance of noise for reducing the impact of the timevarying noise. When this algorithm is used in information fusion, it can effectively overcome the filter divergence, and increase the reliability and accuracy of system. Many research institutions and scholars have applied information fusion technology to the field of mobile robot. Frenchmade Hilare [14] is assembled with vision, sound and laser sensor. Laser sensor and two colour camera are used to find the obstacles in the ALV (Autonomous Land Vehicle) of DARPA (Defense Advanced Research Projects Agency) [15] in America. American-made Pioneer is equipped with sonar ring, laser rangefinder, vision system, electronic compass and collision bumper, which can realize autonomous navigation through multi-sensor data fusion. However, there are no studies on the information fusion of electronic compass and stereo vision to realize navigation. But with the development of electronic compass technology, its refresh rate and accuracy have been greatly improved with location accuracy within $0.5^{\circ}$, resolution up to $0.1^{\circ}$ and $20 \mathrm{~Hz}$ refresh rate. Compared with gyroscope, it has the advantages of low price and non-cumulative error, so it is fully able to meet the requirements of robot localization. 


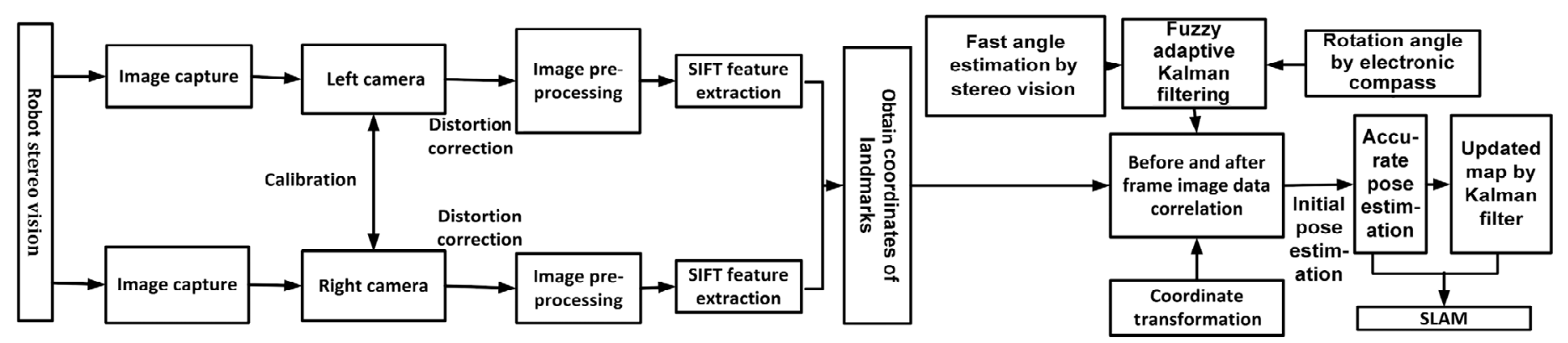

Figure 1. Running processes.

Electronic compass can eliminate the cumulative error of stereo vision-based SLAM [16]. At the same time electronic compass and stereo vision are differently subject to environmental interference and can complement each other's advantages. In this paper, a new fast estimation algorithm of robot's rotation angle by $3 \mathrm{D}$ coordinates of landmarks based on stereo vision is proposed, meanwhile, the rotation angles obtained by electronic compass and stereo vision respectively are fused by an improved FA-EKF. Then initial pose estimation is obtained by the rotation angles and $3 \mathrm{D}$ coordinates of the time $t$ and $t+1$, and accurate pose estimation is realized by an adaptive particle filter. At last, the map is updated by the Kalman filter. Experiment results show that the location accuracy, robustness and real-time performance are better than stereo vision alone and the traditional FA-EKF.

\section{The Proposed Method}

At first, the environmental landmarks based on stereo vision are extracted and matched, 3D coordinate values are obtained by parallax, and the rotation angles obtained by electronic compass and stereo vision respectively are fused by the improved FA-EKF. Then initial pose estimation is obtained by the rotation angles and 3D coordinates of the time $t$ and $t+1$, and accurate pose estimation is realized by an adaptive particle filter to improve the real-time performance. At last, the map is updated by the Kalman filter. The running process is shown in Fig. 1.

\subsection{Initial Pose Estimation}

The initial pose is estimated by electronic compass and stereo vision. The rotation angle is obtained by data fusion between that of electronic compass and that of stereo vision with the improved FA-EKF. If the value of innovation is normal in the adaptive Kalman filter process, the parameters of the filter do not have to be adjusted. If exception but does not exceed a preset limit, the filtering parameters are adjusted. If it exceeds a preset limit, we determine whether electronic compass or stereo vision is invalid according to robot motion characteristics of inertia, historical data and control commands. A fast rotation angle of robot based on the $3 \mathrm{D}$ coordinates of landmarks is proposed in order to improve the real-time performance. As the angle increment of electronic compass is the difference between absolute rotation angles, there

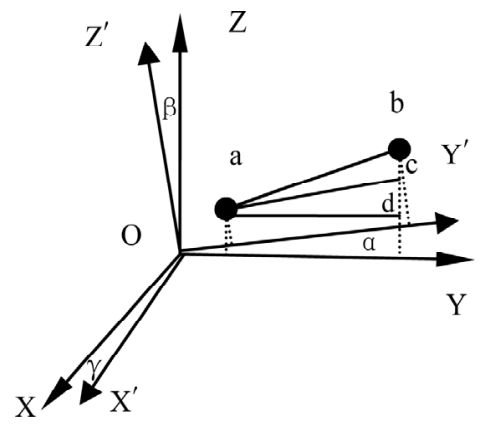

Figure 2. Rotating coordinate diagram.

is no cumulative error. But it is poor against magnetic interference. While stereo vision is affected by light change easily, so, it is complementary between them.

\subsubsection{Fast Estimation of Robot Rotation Angle Based on $3 D$ Coordinates of Stereo Vision Landmarks}

Robot pose changes include rotation and translation. The most common solving method of rotation vector is four elements. However the calculating quantity of four elements is large and the solving of rotation angle is difficult. In order to improve the timeliness, a fast estimation method of rotation angle is put forward in this paper, the principle of which is that the angle change of the line by the two stationary landmarks between the time $t$ and the time $t+1$ and the rotation angle change of robot itself at the time $t$ and the time $t+1$ is basically the same, because the rotation angle change of robot at the time $t$ and the time $t+1$ is small generally and landmarks are still, meanwhile, translation has no effect on rotation angle.

As shown in Fig. 2, the rotation angles between $\mathrm{O}-\mathrm{X}^{\prime} \mathrm{Y}^{\prime} \mathrm{Z}^{\prime}$ and $\mathrm{O}-\mathrm{X} \mathrm{YZ}$ are $\alpha, \beta, \gamma$. The landmark $\boldsymbol{a}$ and the landmark $\boldsymbol{b}$ are two relatively static two points in space. The coordinates of landmark $\boldsymbol{a}$ in O-XYZ and $\mathrm{O}-\mathrm{X}^{\prime} \mathrm{Y}^{\prime} \mathrm{Z}^{\prime}$ are $\left(x_{a}, y_{a}, z_{a}\right)$ and $\left(x_{a}^{\prime}, y_{a}^{\prime}, z_{a}^{\prime}\right)$ respectively. The coordinates of landmark $\boldsymbol{b}$ in $\mathrm{O}-\mathrm{XYZ}$ and $\mathrm{O}-\mathrm{X}^{\prime} \mathrm{Y}^{\prime} \mathrm{Z}^{\prime}$ are $\left(x_{a}, y_{a}, z_{a}\right)$ and $\left(x_{a}^{\prime}, y_{a}^{\prime}, z_{a}^{\prime}\right)$ respectively. Detailed calculation steps are as follows.

Step 1. Initial estimation

Taking the calculation of rotate angle $\alpha$ about $\mathrm{X}$ axis as the example, at first, included angle $\alpha^{\prime}$ between $\mathrm{Y}$ axis and projection of line $\boldsymbol{a} \boldsymbol{b}$ on ZOY plane and included angle $\alpha^{\prime \prime}$ between $\mathrm{Y}$ axis and projection of line $\boldsymbol{a} \boldsymbol{b}$ on $\mathrm{Z}^{\prime} \mathrm{O}^{\prime} \mathrm{Y}^{\prime}$ 
Table 1

Angle Estimation Value

\begin{tabular}{|l|l|l|l|l|l|l|l|l|l|}
\hline \multirow{2}{*}{ Angle } & \multicolumn{3}{|c|}{ The First Group } & \multicolumn{3}{c|}{ The Second Group } & \multicolumn{3}{c|}{ The Third Group } \\
\cline { 2 - 11 } & $\alpha\left(1^{\circ}\right)$ & $\beta\left(2^{\circ}\right)$ & $\gamma\left(3^{\circ}\right)$ & $\alpha\left(6^{\circ}\right)$ & $\beta\left(7^{\circ}\right)$ & $\gamma\left(5^{\circ}\right)$ & $\alpha\left(4^{\circ}\right)$ & $\beta\left(6^{\circ}\right)$ & $\gamma\left(8^{\circ}\right)$ \\
\hline Initial estimation & 0.728 & 2.034 & 2.981 & 4.965 & 7.197 & 4.706 & 2.931 & 6.397 & 7.868 \\
\hline Precise estimation & 0.999 & 1.999 & 3.00 & 5.985 & 7.009 & 4.995 & 3.993 & 6.002 & 7.999 \\
\hline
\end{tabular}

plane are calculated, then the estimation value of rotation angle $\hat{\alpha}$ around $\mathrm{X}$ axis is $\alpha^{\prime}-\alpha^{\prime \prime}$. In the same way, the estimation values of rotation angle $\hat{\beta}$ around $\mathrm{Y}$ axis and $\hat{\gamma}$ around $\mathrm{Z}$ axis are calculated. But, they are mutual coupling because there are rotation motions about three directions. So, the rotation angles are only the preliminary estimate values.

Step 2. Precise estimation

According to the first step analysis, there are large errors between actual value and calculated value because of the influence of the other two rotation angles. So, the precision of the estimate is improved greatly by reducing the influence of the other two angles. Taking the calculation of rotate angle $\alpha$ about $\mathrm{X}$ axis as the example, the new coordinate system $\mathrm{O}-\mathrm{X}^{\prime \prime} \mathrm{Y}^{\prime \prime} \mathrm{Z}^{\prime \prime}$ can be obtained by the backward rotation $\mathrm{O}-\mathrm{X}^{\prime} \mathrm{Y}^{\prime} \mathrm{Z}^{\prime}$ around $\mathrm{Y}^{\prime}$ axis and $\mathrm{Z}^{\prime}$ axis for $\hat{\beta}$ and $\hat{\gamma}$. The angles between $\mathrm{O}-\mathrm{X}^{\prime \prime} \mathrm{Y}^{\prime \prime} \mathrm{Z}^{\prime \prime}$ and $\mathrm{O}-\mathrm{X}^{\prime} \mathrm{Y}^{\prime} \mathrm{Z}^{\prime}$ around $\mathrm{Y}^{\prime}$ axis and $\mathrm{Z}^{\prime}$ axis are very small and negligible, $\alpha$ is recalculated in $\mathrm{O}-\mathrm{X}^{\prime \prime \prime} \mathrm{Y}^{\prime \prime \prime} \mathrm{Z}^{\prime \prime \prime}$ according to the first step method. In the same way, the new coordinate system $\mathrm{O}-\mathrm{X}^{\prime \prime \prime} \mathrm{Y}^{\prime \prime \prime} \mathrm{Z}^{\prime \prime \prime}$ is obtained by the backward rotation $\mathrm{O}-\mathrm{X}^{\prime} \mathrm{Y}^{\prime} \mathrm{Z}^{\prime}$ around $\mathrm{X}^{\prime}$ axis and $\mathrm{Z}^{\prime}$ axis for $\alpha$ and $\hat{\gamma}, \beta$ is recalculated in $\mathrm{O}-\mathrm{X}^{\prime \prime \prime} \mathrm{Y}^{\prime \prime \prime} \mathrm{Z}^{\prime \prime \prime}$, and the new coordinate system $\mathrm{O}-\mathrm{X}^{\prime \prime \prime} \mathrm{Y}^{\prime \prime \prime} \mathrm{Z}^{\prime \prime \prime}$ is obtained by the backward rotation $\mathrm{O}-\mathrm{X}^{\prime} \mathrm{Y}^{\prime} \mathrm{Z}^{\prime}$ around $\mathrm{X}^{\prime}$ axis and $\mathrm{Y}^{\prime}$ axis for $\alpha$ and $\beta, \gamma$ is recalculated in $\mathrm{O}-\mathrm{X}^{\prime \prime \prime} \mathrm{Y}^{\prime \prime \prime} \mathrm{Z}^{\prime \prime \prime}$.

Step 3. If need to further improve the accuracy, repeat Step 2.

Simulation experiment results based on MATLAB7.0 are shown in Table 1. The errors are under $0.1 \%$ and the run time is 240 times four element method in the same environment.

\subsubsection{Rotation Angle Fusion Based on an Improved $F A-E K F$}

The rotation angles obtained by electronic compass and stereo vision respectively are fused by an improved FA-EKF. The improved FA-EKF theory is introduced as follows.

The state predictions based on Kalman filter are given by

$$
\begin{aligned}
K_{k \mid k-1} & =F_{k} X_{k-1 \mid k-1}+B_{k} U_{k} \\
P_{k \mid k-1} & =F_{k} P_{k-1 \mid k-1} F_{k}^{T}+Q_{k} U_{t}\left(\sum_{m}^{-1}+\sum_{t}^{-1}\right)^{-1}
\end{aligned}
$$

the state updating is given by

$$
\begin{aligned}
X_{k \mid k} & =X_{k \mid k-1}+K_{k}\left(Z_{k}-H_{k} X_{k \mid k-1}\right) \\
P_{k \mid k} & =\left(I-K_{k} H_{k}\right) P_{k \mid k-1}
\end{aligned}
$$

and Kalman filtering gain is

$$
K_{k}=P_{k \mid k-1} H_{k}^{T}\left(H_{k} P_{k \mid k-1} H_{k}^{T}+R_{k}\right)^{-1}
$$

where $X_{k \mid k-1}$ is the predicted value; $P_{k \mid k-1}$ is the covariance of the predicted value; $X_{k \mid k}$ is the estimation value of the state updating; $Z_{k}$ and $R_{k}$ are the observed value and its probability; and $F_{k}, B_{k}$ and $H_{k}$ are the state transfer matrix, the control matrix and observation matrix, respectively. This system may be regarded as a constant with time, so let $F_{k}=B_{k}=H_{k}=\mathrm{I}$, the standard Kalman filter output is given by

$$
\begin{gathered}
X_{k \mid k}=P_{k \mid k}\left(Q_{k}^{-1} X_{k \mid k-1}+R_{k}^{-1} Z_{k}\right) \\
P_{k \mid k}=\left(Q_{k}^{-1}+R_{k}^{-1}\right)^{-1} \\
v(k)=Z_{k}-X_{k \mid k-1} \\
S(k+1)=Q_{k}+R_{k}
\end{gathered}
$$

where $X_{k \mid k-1}$ and $Q_{k}$ are the observations obtained by electronic compass and its covariance matrix, respectively; $Z_{k}$ and $R_{k}$ are the observations with stereo vision and its covariance matrix; $X_{k \mid k}$ is the estimation value of the rotation angle updated; $P_{k \mid k}$ is the covariance matrix of the rotation angle updated; $v(k)$ is the innovation; and $S(k+1)$ is the covariance matrix of innovation.

Because the establishments of accurate statistical characteristics of system noise and measurement noise are very difficult, in order to solve this problem, the fuzzy adaptive filtering is adopted in the paper. The noise model is revised by

$$
\begin{aligned}
& Q_{k}=a(k) Q \\
& R_{k}=b(k) R
\end{aligned}
$$

where $\mathrm{Q}$ and $\mathrm{R}$ are the initial noise models; $a(k)$ and $b(k)$ are the on-line adjusting weights. The covariance matrix of innovation is revised by $S(k+1)=a(k) Q_{k}+b(k) R_{k}$. 

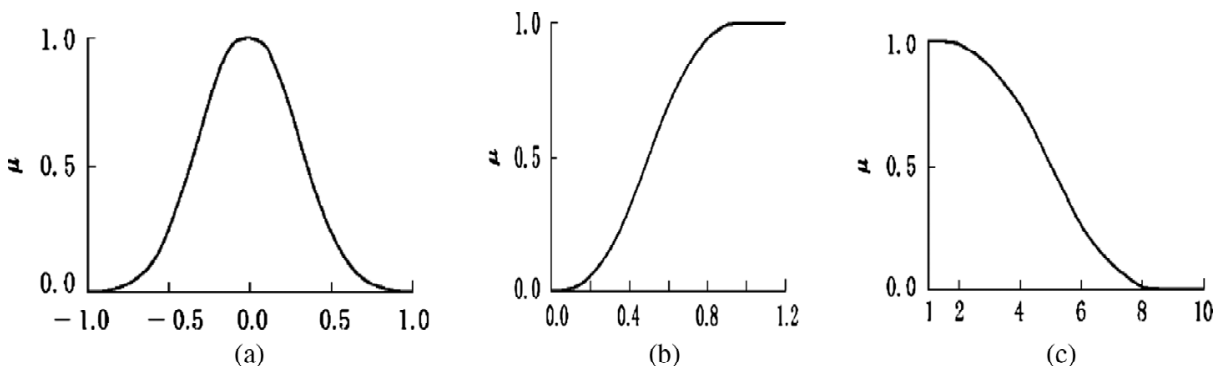

Figure 3. Fuzzy membership function: (a) П-type membership function; (b) S-type membership function; and (c) Z-type membership function.

Based on the above discussion, Kalman filter based on fuzzy logic structure is proposed with fuzzy mapping between the innovation model and noise model. The on-line monitoring of the filter's stability is realized by the statistical characteristics' changes of innovations. When the filter is not stable, the weights of system noise and measurement noise are changed by $a(k)$ and $b(k)$. The sample mean value of innovation's mean square value and variance within a certain time window $M$. Actual mean and variance of the innovation at each moment are defined by

$$
\begin{gathered}
\bar{v}(k)=\frac{1}{M} \sum_{i=k-M+1}^{k} v(i) v(i)^{T} \\
\bar{S}(k)=\frac{1}{M} \sum_{i=k-M+1}^{k} S(i) S(i)^{T}
\end{gathered}
$$

where $M$ is a certain time window. If $v(k) v(k)^{T} \leq s(\bar{v}(k))$, the filter is in the normal work and unnecessary to adjust, avoiding frequent adjustment to reduce the real-time. If $s\left(\bar{v}(k)<v(k) v(k)^{T} \leq n(\bar{v}(k))\right.$, the filter is in the abnormal and noise characteristic is adjusted. If $n(\bar{v}(k))<v(k) v(k)^{T}$, some sensor is invalid and its datum are not available; $s$ and $n$ are the adjusting coefficients, and $n \geq s \geq 1$. The confidences of innovation's mean and variance are given by

$$
\begin{aligned}
& q_{1}(k)=\bar{v}(k) / \bar{z}_{k} \\
& q_{2}(k)=\operatorname{tr} \bar{S}(k) / \operatorname{tr} S(k)
\end{aligned}
$$

The fuzzy membership's matches of in-out variables are adopted in the paper in order to avoid fuzzy reasoning and to improve the real-time. Fuzzy membership functions are shown in Fig. 3. The calculations of $a(k)$ and $b(k)$ are given by

$$
\begin{aligned}
a(k) & =1+\eta\left[F_{\Pi}\left(q_{1}(k)+F_{s}\left(q_{2}(k)\right) / 2\right]\right. \\
b(k) & =\left[F_{\Pi}\left(q_{1}(k)+F_{z}\left(q_{2}(k)\right)\right] / 2\right]
\end{aligned}
$$

where $F_{\Pi}$ is $\Pi$-type Gaussian fuzzy membership function; $F_{s}$ is S-type fuzzy membership function; $F_{z}$ is Z-type fuzzy membership function; and $\eta$ is constant.

\subsubsection{Calculation of Translation Increment}

Let matching landmark sets of the before and after frames be $X_{t}^{i}$ and $X_{t+1}^{i}$

$$
\begin{gathered}
c_{t}=\frac{1}{N} \sum_{i=1}^{N} X_{t}^{i} \\
c_{t+1}=\frac{1}{N} \sum_{i=1}^{N} X_{t+1}^{i}
\end{gathered}
$$

where $c_{t}$ and $c_{t+1}$ are the centroids of the before and after frames. The translation increment of robot pose is obtained by

$$
t=c_{t}-R c_{t+1},
$$

where $\boldsymbol{R}$ is the rotation matrix and obtained by the rotation angles with information fusion.

\subsection{Accurate Location based on Adaptive Particle Filter}

\subsubsection{The Principle of Adaptive Particle Filter}

To improve estimation accuracy of robot pose, accurate pose estimation based on adaptive particle filtering is adopted in the paper. Compared with EKF (Extended Kalman filtering) and UKF (Unscented Kalman filtering), adaptive particle filtering has higher accuracy. Compared with traditional particle filtering, adaptive particle filtering has higher real-time performance because the particle number of traditional particle filtering is changeless, which can adaptively adjust particle number through the prediction of error. When the errors between the predicted values and the observed values exceed the specified values adaptive particle filtering enlarges the scope of sampling and particle number, and it works the other way as well. The relationship between particle number and the errors is expressed as

$$
\begin{gathered}
N=\left(M_{\max }-M_{\min }\right) \times \frac{e_{\text {current }}}{E_{\max }}+M_{\min }, \\
e_{\text {current }}=\frac{1}{k} \sum_{i=1}^{k}\left(z_{t}^{i}-m_{t}^{i}\right),
\end{gathered}
$$




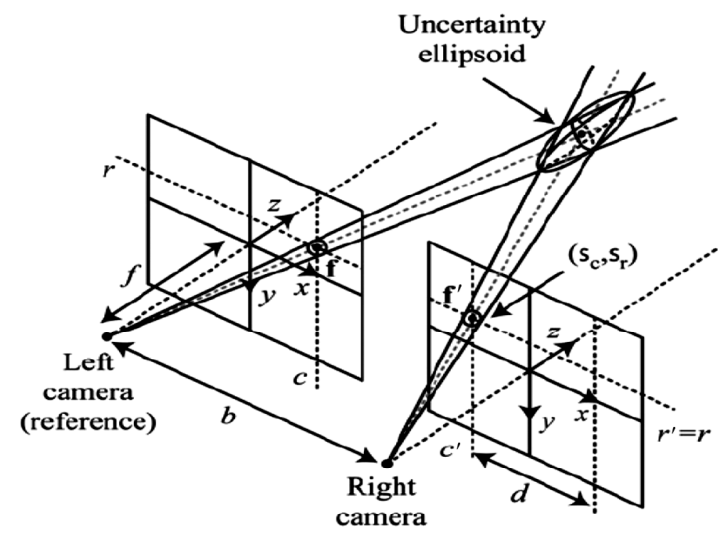

Figure 4. Uncertainty configuration of a 3D landmark.

where $k$ is the current landmark number; $z_{t}^{i}$ is the observed value of landmark based on stereo vision; $m_{t}^{i}$ is the predicted value of landmark. Let $E_{\max }=60, M_{\max }=100$, $M_{\min }=50$.

\subsubsection{Realized Steps of Robot Localization Based on Adaptive Particle Filter}

There are 7 steps, and detailed calculation steps are as follows.

Step 1. Pose particle sampling

The initial pose is sampled by Sequential Importance Sampling - SIS.

Step 2. Calculation of landmark prediction value

The landmarks are obtained by stereo matching of SIFT (Scale Invariant Feature Transform) feature points, "m" is defined as the landmark set, let $\mathrm{m}=\left\{\mathrm{m}^{j}\right\}, \mathrm{j}=\{1, \ldots$, $\mathrm{M}\}$. The calculation of the landmark prediction value is calculated by

$$
\bar{m}_{i}^{j}=R^{i} X^{j}+T^{i}
$$

where $R^{i}$ and $T^{i}$ are relative rotation matrix and translation vector of each pose particle in the global coordinate system respectively; and $X^{i}$ is the $3 \mathrm{D}$ coordinates of each landmark in the global coordinate system; and $\bar{m}_{i}^{j}$ is the prediction value of each landmark.

Step 3. Calculation of landmark observation value

As shown in Fig. 4, the landmark observation value is calculated by

$$
X=\left(c-c_{0}\right) \frac{b}{d} ; Y=\left(r-r_{0}\right) \frac{b}{d} ; Z=f \frac{b}{d},
$$

where $r$ and care the projection coordinates of the left SIFT feature points, $\mathrm{r}_{0}$ and $\mathrm{c}_{0}$ are the central coordinates of the left image.

Step 4. Particle weight calculation and normalization processing

The particle weight is obtained by the probability of each particle. For the error distribution of each landmark is independent, the probability of each particle is given by

$$
p\left(P \mid h_{i}\right)=\prod_{i} p\left(P_{i} \mid h_{i}\right)
$$

where $p\left(P_{i} \mid h_{i}\right)$ shows the influence of each landmark $P_{i}$ on each particle $h_{i}$, so the calculation of $p\left(P_{i} \mid h_{i}\right)$ is the key in particle weight calculation. Assuming $p\left(P_{i} \mid h_{i}\right)$ obeys the Gaussian distribution for calculation simpleness, its mean value is the difference between the observation value of landmark and the prediction value, and its covariance is the sum of the observation value's covariance and prediction value's covariance. Detailed calculations are shown in [16].

$$
\begin{aligned}
p\left(P_{i} \mid h_{i}\right)= & N\left(\bar{P}_{i}-\bar{m}_{i}, \sum_{\bar{P}_{i}}+\sum_{\bar{m}_{i}}\right) \\
= & \left(2 \pi\left|\sum_{\bar{P}^{i}}+\sum_{\bar{m}^{i}}\right|\right)^{\frac{1}{2}} \\
& \times \exp \left\{-\frac{1}{2}\left(\bar{P}_{i}-\bar{m}_{i}\right)^{T}\left(\sum_{\bar{P}_{i}}+\sum_{\bar{m}_{i}}\right)^{-1}\right. \\
& \left.\left(\bar{P}_{i}-\bar{m}_{i}\right)\right\}
\end{aligned}
$$

Where $\bar{P}_{i}$ is the observation value of each landmark and $\bar{m}_{i}$ is the prediction value, and $\sum_{\bar{P}_{i}}$ is the observation value's covariance and $\sum_{\bar{m}_{i}}$ is the prediction value's covariance. The probability of each particle under all effects of landmarks is easily calculated by expression (22). The particle weight calculation is given by

$$
\omega_{t}^{[k]}=\omega_{t-1}^{[k]} p\left(P_{t} \mid h_{t}^{[k]}\right)
$$

where $\omega_{t}^{[k]}$ is the weight of pose particle at time $t$, and $\omega_{t-1}^{[k]}$ is the weight of pose particle at time $t-1$. The normalized weight is given by

$$
\bar{\omega}_{t}^{[k]}=\frac{\omega_{t}^{[k]}}{\sum_{k=1} \omega_{t}^{[k]}}
$$

Step 5. Calculation of the adaptive sampling particle number and particle resampling

Calculation of the adaptive sampling particle number is shown in (19) and particle self-adaption resampling algorithm is adopted in particle resampling.

Step 6. Precise estimation of robot pose

Precise estimation of pose and covariance is calculated by

$$
\hat{x}_{t}=\sum_{i=1} \bar{\omega}_{t}^{i} x_{t}^{i}
$$

$$
P_{t}=\frac{1}{N} \sum_{i=1}^{N}\left(\hat{x}_{t}-x_{t}^{i}\right)\left(\hat{x}_{t}-x_{t}^{i}\right)^{T}
$$

Step 7. Map management

The map is built by the matched SIFT features in this paper, which entails the insertion, update and deletion of landmarks (matched SIFT features). The map management can be summarized by this sequence of steps.

(1) The landmarks in the map without a correspondence are not modified. Landmarks which have not been observed in a significant number of times are deleted from the map, as they are considered as non-stable. 


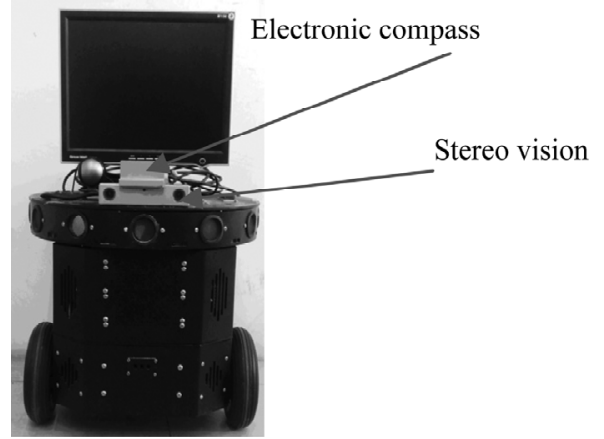

Figure 5. The experiment platform.

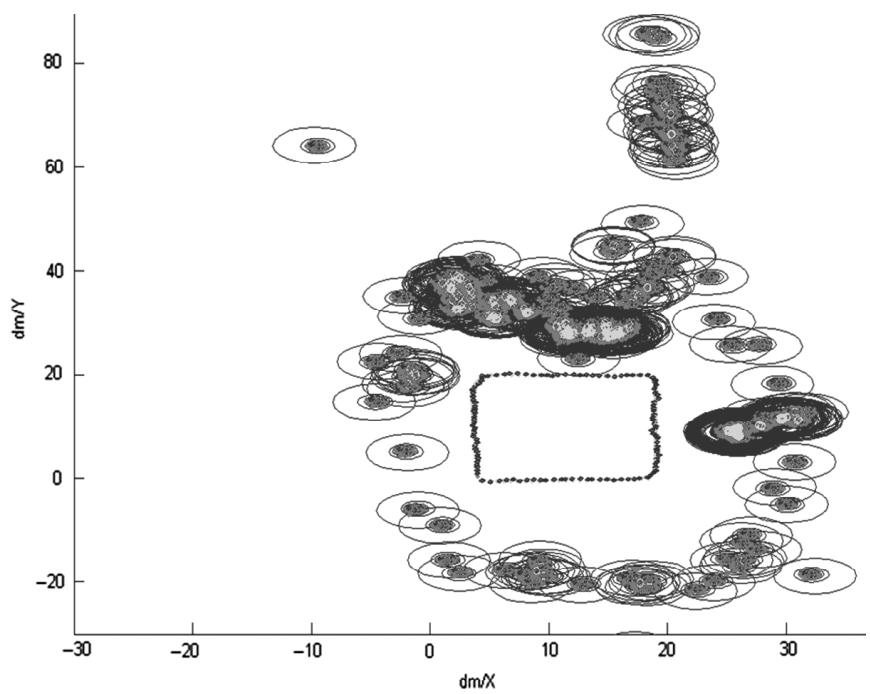

Figure 6. Robot SLAM based on information fusion.

(2) The observed landmarks with no correspondences are introduced into the map.

(3) The positions of the landmarks in the map with a correspondence are updated with Kalman filtering.

Let $X_{k \mid k-1}=\bar{m}_{t}^{i}, Q_{k}=\sum_{\bar{m}}, X_{k \mid k}=\mu_{m}, P_{k \mid k}=\sum_{m}$, $Z_{k}=\bar{z}_{t}^{i}, R_{k}=\sum_{t} ;(28)$ and (29) are obtained by (4) and (5).

$$
\begin{gathered}
\mu_{m}=\sum_{m}\left(\sum_{\bar{m}}^{-1} \bar{m}_{t}^{i}+\sum_{t}{ }^{-1} \bar{z}_{t}^{i}\right) \\
\sum_{m}=\left(\sum_{\bar{m}}^{-1}+\sum_{t}^{-1}\right)^{-1}
\end{gathered}
$$

In (28) and (29), $\mu_{m}$ and $\sum_{m}$ are updated estimated value and covariance. Predicted value $\bar{m}_{t}^{i}$ and observed value $\bar{z}_{t}^{i}$ are obtained by Step 1 and Step 2 in the paper, the covariance $\sum_{\bar{m}}$ of Predicted value and the covariance $\sum_{t}$ of observed value are calculated with the principle of error propagation of the first-order Taylor expansion [18].

\section{Experimental Studies}

As shown in Fig. 5, the experiment platform includes Bumblebee2 Camera which is a binocular stereo vision

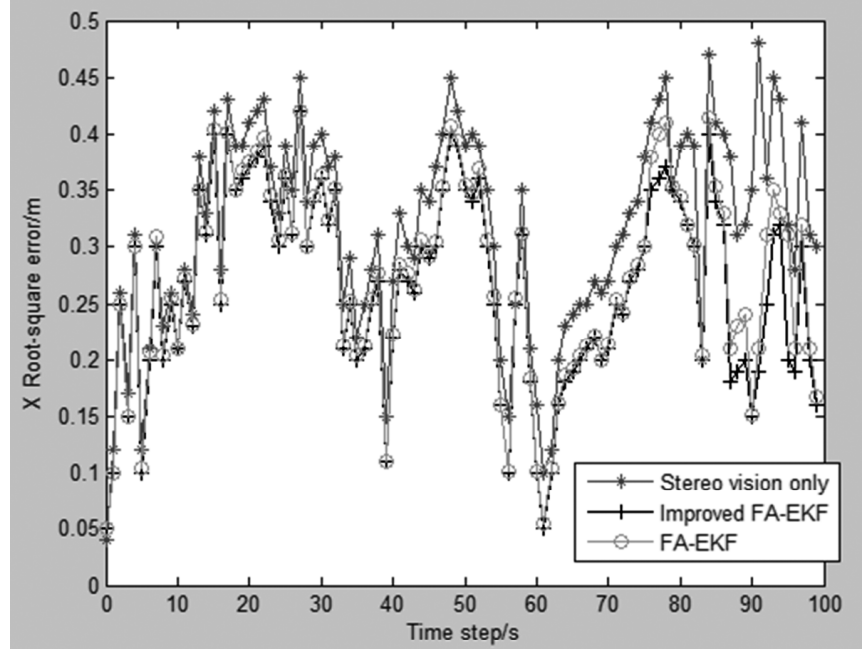

Figure 7. $\mathrm{X}$ axis direction estimation error.

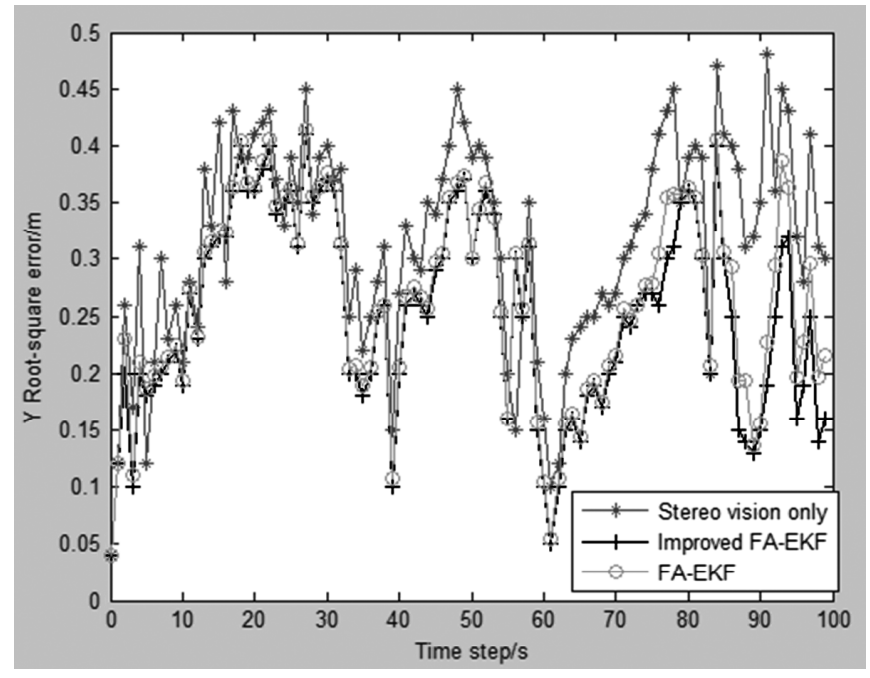

Figure 8. Y axis direction estimation error.

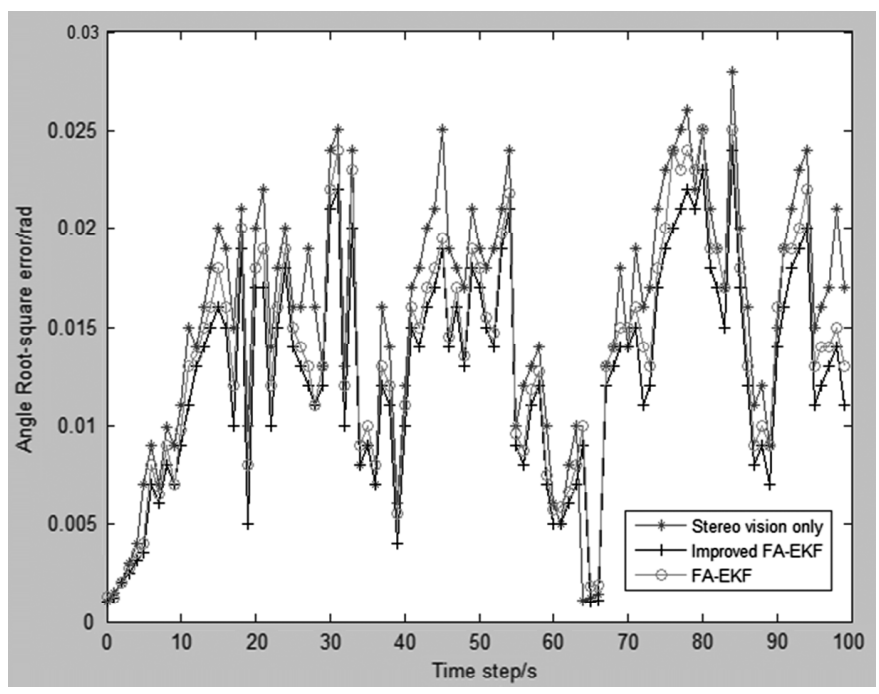

Figure 9. Angle estimation error.

made by Point Grey Research company and the electronic compass LP3300 and the mobile robot AS-R made by ShangHai x-partner company. The image size is $640^{*} 486$. The hardware platform includes Intel(R) Core(TM)2 Duo 
Table 2

Algorithm Comparison

\begin{tabular}{|l|c|c|c|c|}
\hline & X RMSE $(\mathrm{m})$ & Y RMSE $(\mathrm{m})$ & Angle RMSE (rad) & Runtime of Each Step (ms) \\
\hline Improved FA-EKF & 0.179 & 0.202 & 0.0148 & 103 \\
\hline Stereo vision only & 0.211 & 0.243 & 0.0171 & 131 \\
\hline FA-EKF & 0.184 & 0.208 & 0.0151 & 118 \\
\hline
\end{tabular}

CPU E7200 @ 2.53G Hz and memory 1G. Let the robot motion environment: indoor flat in $50 \mathrm{~Hz} 220 \mathrm{~V} \mathrm{AC}$ environment, uneven illumination, line speed: $0.1 \mathrm{~m} / \mathrm{s}$ and maximum angular velocity: $0.3 \mathrm{rad} / \mathrm{s}$. The pose estimation and map update of robot based on information fusion in the paper are shown in Fig. 6, which shows the uncertainties of landmarks reduce gradually through update with $\mathrm{KF}$ during robot movement. In order to verify integrated location algorithm in this paper, the location accuracy is compared with mainstream SLAM method [16] and mainstream fusion method [17]. The mainstream SLAM method [16] realizes SLAM with stereo vision only, and the mainstream fusion method [17] is traditional FA-EKF algorithm. The $\mathrm{x}$ axis location error is shown in Fig. 7, the $\mathrm{Y}$ axis location error is shown in Fig. 8 and the angle of rotation error is shown in Fig. 9. The performance parameters of three algorithms are shown in Table 2. Experiment results show that the location accuracy and robustness and real-time performance of integrated location algorithm are better, because the information fusion in this paper decreases significantly the effects of uneven illumination and electromagnetic interference, and because the fast estimation method of robot rotation angle and improved FA-EKF algorithm and adaptive particle filter algorithm reduce the computation complexity.

\section{Conclusion}

In this paper we have addressed SLAM problems for stereo vision and electronic compass system based on each other's advantages. The novel estimation method of robot rotation angle based on 3D coordinates of stereo vision landmarks is fast and simple. The improved FA-EKF and adaptive particle filter are used to achieve information fusion of two sensors' datum. Experiment results show that the location accuracy, the robustness and the real-time performance are better than stereo vision alone and the traditional FA-EKF.

\section{Acknowledgement}

This work is supported by the Spring Plan of Ministry of Education of China (Z2012014), the Key scientific research fund of Xihua University (Grand No:z1420210), and the Open Research Fund of Key Laboratory of Manufacture and Automation Laboratory (Xihua University, szjj2015082).

\section{References}

[1] Z.W. Liang and Y.Y. Chen, Just-in-time cooperative simultaneous localization and mapping: A robust particle filter approach, International Journal of Robotics \& Automation, 39(2), 2014, 155-161.

[2] S. Sajad, P. Liam, T. Michael, and T. Michael, Occupancy grid map merging for multiple robot simultaneous localization and mapping, International Journal of Robotics \& Automation, 30(2), 2015, 149-157.

[3] F. Auat Cheein, G. Steiner, G. Perez Paina, and R. Carelli, Optimized EIF-SLAM algorithm for precision agriculture mapping based on stems detection, Computers and Electronics in Agriculture, 78(2), 2011, 195-207.

[4] V. Ila, M. Josep Porta, and A. Juan, Amortized constant time state estimation in Pose SLAM and hierarchical SLAM using a mixed Kalman-information filter, Robotics and Autonomous Systems, 59(5), 2011, 310-318.

[5] H. Kwon, M. Khalil, A. Yousef, and C. Kak, Building 3D visual maps of interior space with a new hierarchical sensor fusion architecture, Robotics and Autonomous Systems, 61(8), 2013, 749-767.

[6] L. Paz, J. Tardos, and J. Neira, Divide and conquer: EKF SLAM in o (n), IEEE Trans Robot, 24(5), 2008, 1107-1120.

[7] B. Khaleghi, A. Khamis, and F.O. Karray, Multisensor data fusion: A review of the state-of-the-art, Information Fusion, 14(1), 2013, 28-44.

[8] X.G. Jian, H.S. Jia, and L.D. Shi, Advances on multi-sensor information fusion technologies, Chinese Journal of Construction Machinery, 7(2), 2009, 227-232.

[9] X.D. Li, J. Dezert, and C.F. Smarandache, Evidence supporting measure of similarity for reducing the complexity in information fusion, Information Sciences, 181(1), 2011, 1818-1835.

[10] Z.G. Liu, D. Jean, and Q. Pan, Combination of sources of evidence with different discounting factors based on a new dissimilarity measure, Decision Support Systems, 52(1), 2011, 133-141.

[11] A.L. Jousselme and P. Maupin, Distances in evidence theory: Comprehensive survey and generalizations, International Journal of Approximate Reasoning, 53(1), 2012, 118-145.

[12] Y. He, L.F. Hu, and X. Guan, New method for measuring the degree of conflict among general basic probability assignments, Science China: Information Sciences, 55(2), 2012, 312-321.

[13] X.J. Shen, Y.T. Luo, and Y.M. Zhu, Globally optimal distributed Kalman filtering fusion, Science China: Information Science, 55(3), 2012, 512-529.

[14] W.E. Grimson, Model-based recognition and location from sparse range or tactile data, Robotics Research, 3(3), 1984, $3-35$.

[15] M. Daily, J. Harris, and D. Keirsey, Autonomous cross-country navigation with the ALV, Proceedings of IEEE Conference on Robotics and Automation, 1988, 718-726.

[16] F.A. Moreno, J.L. Blanco, and J. Gonzalez, Stereo vision specific models for particle filter-based SLAM, Robotics and Autonomous Systems, 57(1), 2009, 955-970.

[17] Mei Jin, Jinge Zhao, Ju Jin, Guohui Yu, and Wenchao Li, The adaptive Kalman filter based on fuzzy logic for inertial motion capture system, Measurement, 49(3), 2014, 196-204.

[18] J. M. Li, J.G. Wang, and W.T. Zhou, Research on information technology with robot pose estimation and accuracy analysis based on stereo vision, Proceedings-IEEE 9th International Conference on Mobile Ad-Hoc, Dalian, CA, 2013, 555-559. 


\section{Biographies}

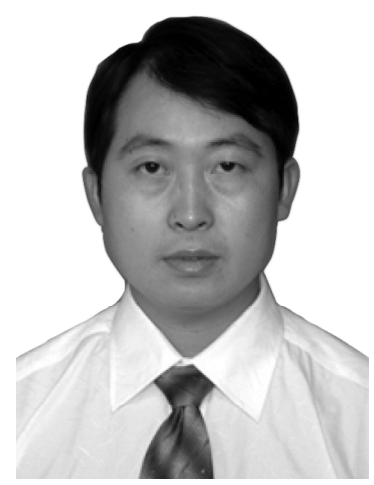

Junmin $L i$, born in 1975 , is currently an associate professor at Xihua University, China, since 2009. He received his Ph.D. degree from Sichuan University, China, in 2014. His research interests include robotics and vision.

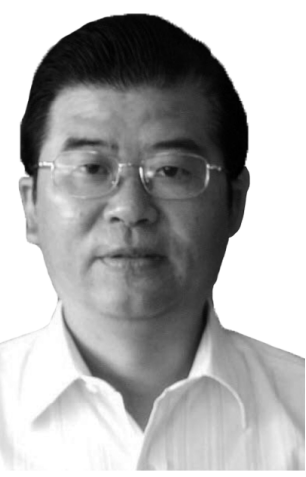

Jinge Wang, born in 1957, is currently a professor at Xihua University, China. He received his Ph.D. degree from Chongqing University, China, in 1992. His research interests include mechanical transmission and robotics.

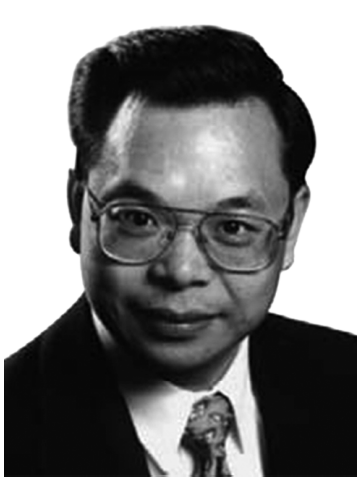

Simon X. Yang is currently a professor at University of Guelph, Canada. He received his Ph.D. degree from the University of Alberta, Canada. His research interests include intelligent systems, robotics, sensors, communications and networking, control systems, vision and signal processing.

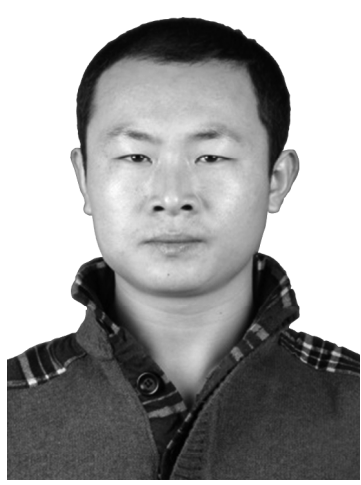

Shiwei Jia, born in 1989, is currently pursuing a postgraduate degree at Xihua University, China. His research interests include robotics. 